\title{
Strengthening the activation of unconsciously activated memories
}

\author{
LEILANI B. GOODMON and DOUGLAS L. NELSON \\ University of South Florida, Tampa, Florida
}

\begin{abstract}
Reading a word activates a set of associated words-for example, reading CAB activates car, driver, and other associates. This research was conducted to determine whether the unconscious activation of these associates adds activation to other associates in the same set. Words were studied, and recall was tested with cues comprised of associates directly activated by the target. On the basis of preexisting links, the associates serving as test cues also received strong (e.g., car) or weak (e.g., driver) additional inputs from other associates in the set. Cues receiving stronger inputs from other associates were more effective, indicating that unconsciously activated associates strengthen one another. This effect declined when conditions disrupted the retrieval of context features linked to the episode. Attending to another task during or after study or receiving implicit test instructions decreased the effects of input from other associates. Importantly, disruptions affected input from other associates more than direct input from the target, suggesting that the influence of context wanes as the links in the network become more distant.
\end{abstract}

Accessing preexisting information is necessary for comprehension (see, e.g., Kintsch, 1988). The priming and memory literatures are converging on the idea that directly experienced concepts activate or access related memories - for example, seeing the word $\mathrm{CAB}$ activates its associates bus, car, driver, and so on. In the priming literature, faster response times to a word's associates than to unrelated words are attributed to automatic forwardacting activation (e.g., Neely \& Durgunoğlu, 1985) or to fast-acting compound cue access (Ratcliff \& McKoon, 1988). In the memory literature, cued recall is more likely to be correct when a studied word activates an associate that becomes its test cue and when its associates are more connected (e.g., Humphreys \& Galbraith, 1975; Nelson \& Zhang, 2000; Tulving \& Thomson, 1973). In addition, false recognition occurs when an unstudied associate of a studied word appears during testing (Underwood, 1965), and false recall occurs when each of a series of studied items activates a word that does not appear in the list (e.g., Roediger \& McDermott, 1995).

This article is centered on the hypothesis that, once activated, the associates of studied words strengthen one another as a function of preexisting links among them. The work reported here differs from past research on associative connectivity in that we are not asking whether activation along the links among the associates strengthens the target. Instead, we determine whether activation

This research was supported by Grant MH16360 from the National Institute of Mental Health to D.L.N. We thank Cathy McEvoy for her comments on an earlier draft. Correspondence concerning this article should be addressed to D. L. Nelson, Department of Psychology, PCD 4118G, University of South Florida, Tampa, FL 33620-8200 (e-mail: dnelson2@chuma1.cas.usf.edu). along the links among the associates strengthens an associate in the target's set. The question is whether the activation of one associate adds activation to another associate when preexisting links bind them together. If one associate gains strength from others, then it will be a more effective test cue for recalling the studied word, or target, which was the word that recruited the set in the first place (Nelson \& Zhang, 2000; Tulving \& Thomson, 1973). Such an effect would reflect the influence of an automatic unconscious process.

Past research indicates that the presence of many preexisting associative links among the associates of a target word facilitate its recall. The effects of such connectivity are relevant because the emphasis in this article will be on the effects of a subset of such links. As is illustrated in Figure 1, the word $\mathrm{CAB}$ has a relatively small set of eight associates, each of which is linked to an average of two other associates (e.g., taxi is linked to car, ride, and driver). The relative strength of each link is indexed independently through free association, but the figure is not drawn to scale and some links have been omitted for simplicity (for the complete network, see Nelson, McEvoy, \& Schreiber, 1999). In experiments on connectivity, study words are selected from a free association database of 5,000 words, so that half have many and half have few preexisting associate-to-associate links. During testing, associated words are presented as novel extralist cues for recalling the studied words. The findings of this cued recall task indicate that studied words with higher levels of associate-to-associate connectivity were more likely to be recalled than those with lower levels.

Connectivity effects have been found for high- and low-frequency words, for words having small associative sets and words having large ones, and for words with 


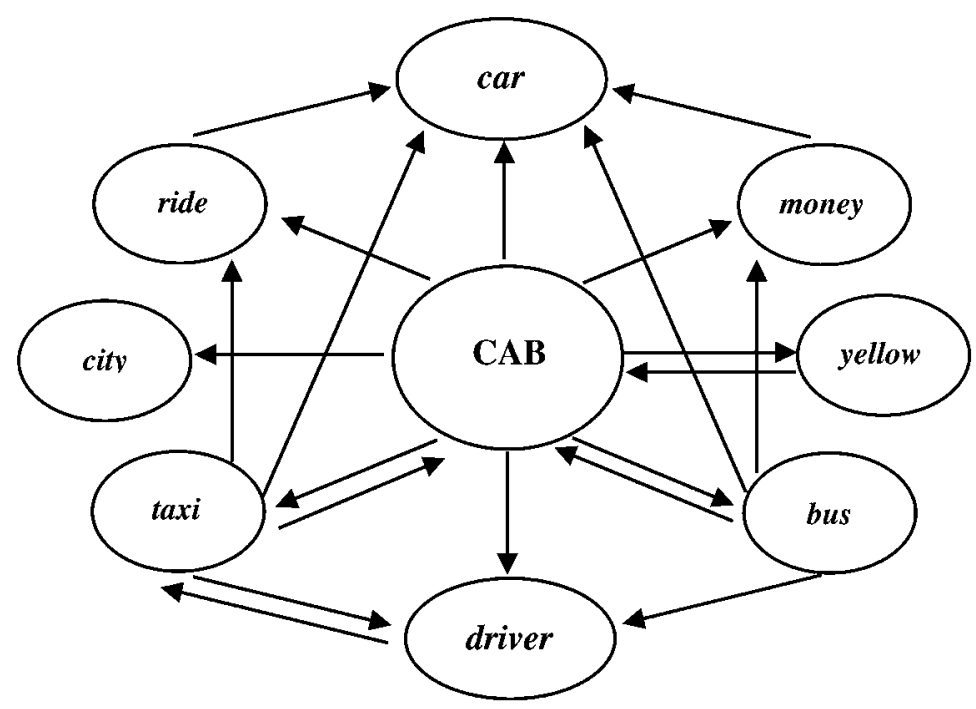

Figure 1. The associative set of a sample target word (CAB) and its binding links.

many or few resonant links back to the target (Nelson, Bennett, Gee, Schreiber, \& McKinney, 1993; Nelson \& Goodmon, 2002; Nelson, McEvoy, \& Pointer, 2003). As is shown in Figure 1, resonant links are associate-to-target links (e.g., taxi to $\mathrm{CAB}$, bus to $\mathrm{CAB}$ ). High connectivity among the target's associates increases target recall for young and old participants, for strong and weak cues, and after short and long study times (Nelson et al., 2003; Nelson, Schreiber, \& McEvoy, 1992). However, the effects of connectivity on target recall are sensitive to disruptions. Such effects are reduced when attention to the encoding context is disrupted during or after study. For example, in comparison with incidental semantic and intentional learning conditions, connectivity effects are reduced under vowel-naming study conditions (Nelson et al., 1993). They are also reduced after participants do math problems immediately after study (Nelson, McKinney, Gee, \& Janczura, 1998).

Connectivity is a global index that refers to links among all associates in the set, and in the present experiments interest is focused on the local effects of input from other associates to the single associate serving as the test cue (called other-associate strength). The distinction is reified in Table 1, which shows an $n \times n$ association matrix for a hypothetical target with four associates. The target produces Associates 1-4 with free association probabilities of $.60, .20, .10$, and .10 . Associate 1 produces the target as an associate at .20, and it produces Associates 3 and 4 at .35 and .05 , respectively. Associate 2 produces Associate 3 at .30, and so on. In this matrix, connectivity refers to the five associate-to-associate links in the set, but note that any one of these associates could serve as the test cue. For example, with Associate 3 selected as the cue, there is input from all three of the other associates in the set, whereas with Associate 4 selected, there is input from only two of the associates. Associate 3 has more input and, according to the free association index, has higher other-associate strength. Granted similar direct input from the target, Associate 3 is a potentially stronger test cue because of input from other members of the target's set. To return to Figure 1, car will be a better cue than driver for $\mathrm{CAB}$, because car receives more input from other associates. An associate will be a stronger test cue only if input from other associates in the target's set adds activation to the strength that it garners from the target. Whether other-associate strength effects are observed is also likely to depend on the encoding and retrieval conditions. Just as global connectivity effects are reduced when memory for context is disrupted, the effects of local other-associate strength are likely to be re-

Table 1

$n \times n$ Association Matrix for a Hypothetical Studied Word and Its Four Associates

\begin{tabular}{lccccc}
\hline & Target & A1 & A2 & A3 & A4 \\
\hline Target (backward strength) & 1.00 & .60 & .20 & $\mathbf{. 1 0}$ & $\mathbf{. 1 0}$ \\
Associate 1 & .20 & & & $\mathbf{. 3 5}$ & $\mathbf{. 0 5}$ \\
Associate 2 & .05 & & &. $\mathbf{3 0}$ & \\
Associate 3 & .05 & & & & $\mathbf{. 0 5}$ \\
Associate 4 & .05 & & & $\mathbf{. 1 5}$ & \\
Other-associate strength & .35 & .00 & .00 & $\mathbf{. 8 0}$ & $\mathbf{. 1 0}$ \\
Net associate strength & 1.35 & .60 & .20 & $\mathbf{. 9 0}$ & $\mathbf{. 2 0}$ \\
\hline
\end{tabular}

Note-A, associate. 
duced by similar disruptions. Here, we test these expectations, but before describing the experiments, we turn to PIER2, the model that was designed to explain the effects that implicitly activated associates have on memory and that motivated the present research (Nelson et al., 2003; Nelson et al., 1998).

PIER2 provides an algorithm for computing the activation strengths of the target and its individual associates (see Appendix A, Equations A1 and A1a for numerical examples). The model assumes that experiencing a word provides immediate parallel access to its associates and the links that bind them together. This activation strengthens the target and its associates and produces an implicit representation that can support recall. Greater numbers and stronger preexisting links among a word's associates add to the strengths of all units comprising its network. The target activation equation computes net strength by summing the strengths of the individual links in the association matrix. Target activation strength is determined by summing over all links - for example, by summing the strengths of all links shown in Table 1. With this rule, target-to-associate, associate-to-associate, and associateto-target links contribute additively to net target activation. Associate activation strength is determined by adding only the specific inputs from the target and from other associates in the set. As is shown in the last row of Table 1, the net strength of Associate 3 is greater than that of Associate 4 , so it is predicted to be a stronger cue.

In PIER2, with all other features equated, associates that receive more activation from other associates in the target's set are predicted to be more effective as cues for recovering the target. This effectiveness, however, depends in part on the recovery of information encoded about context associated with the setting. The model assumes that studying a word produces an implicit representation that encodes the target's activated associative structure. It also assumes that studying a word produces an explicit representation that encodes the results of processing activities, as well as a context representation that encodes source information (see Raaijmakers \& Shiffrin, 1981). Evaluations of the formal computations of the model (e.g., Nelson \& Zhang, 2000) have ignored the influence of context disruptions, but such disruptions can substantially reduce the influence that implicitly activated associates have on recall (Nelson \& Goodmon, 2003; Nelson $\&$ McEvoy, in press). Hence, whether activation received from other associates increases cue effectiveness is expected to depend on retrieval context. The influence of context in an associative network can be implemented formally by weighting each link by a context weight (e.g., by multiplying each link shown in Table 1 by values ranging from 0 to -1.0 ). Weighting target-to-associate backward connections, associate-to-associate connectivity, and associate-to-target resonant links by a constant predicts that disrupting memory for context will have similar effects on each type of link in this model. Assigning different weights to each type of link allows the model to predict that context disruptions will have different effects for different types of links.
The influence of other associate links also depends on PIER2's retrieval process. Retrieval is determined by the intersection of the normative strengths of the test cue and its associates with the primed context-weighted strengths of the target and its associates. Intersections arise from forward cue-to-target links, two-step mediators, primed backward target-to-cue links, and shared associates (see Appendix A, Equations A2 and A3). The intersection of cue and primed target links defines a signal whose retrieval is made relative to the strength of the noise produced by competing associates activated by the cue and by the target. In the model, associates of the target and of the test cue either help or hinder recall depending on whether they link the cue with the target or provide competition. The result of these calculations is a probability that a specific cue will produce the recovery of a specific target.

This model successfully predicts cued recall for thousands of word pairs (Nelson et al., 2003; Nelson \& Zhang, 2000). PIER2's calculations (Equation A1a) predict that associates of the target used subsequently as test cues will vary in effectiveness in accordance with how strongly they are activated by other associates in the set. Those that receive more activation from other associates are predicted to be better cues. This prediction was evaluated in both Experiments 1 and 2, and in addition, both experiments addressed the issue of context disruptions. In Experiment 1 , memory for context was disrupted with two different manipulations, one during and one after study. The disruption during study required participants to say the word and then name its vowels. Vowel naming is regarded as a disruption because it represents an unusual word processing task that diverts attention away from meaning and away from treating the word as a unit. In the nondisruption conditions, participants named the study words and then rated them for pleasantness or tried to remember as many as possible. These two conditions are considered nondisruptive because participants are asked to focus on meaning or to learn the list words in ways that are compatible with normal processing, such as rehearsal. The disruption of attention after study was implemented by testing cued recall immediately or after 10 min of multiplication. The math task represents a disruption with respect to the list processing because it diverts attention to a conceptually different procedure. PIER2 predicts that all of these disruptions will reduce access to what was activated during study by reducing the likelihood that context information linked to the processing of the target will be retrieved. Just as global connectivity effects are reduced by disruptions, the model predicts that other-associate strength effects will be reduced.

In Experiment 2, other-associate strength and backward strength are crossed in a factorial design. As is shown in Table 1, these two sources of strength are added together to produce net associate strength. For example, for Associate 3 a backward strength of . 10 is added to an otherassociate strength of .80 to produce a net strength of .90 . PIER2 predicts that increases in backward and otherassociate strength will facilitate explicit cued recall be- 
cause the activation strength of the test cue increases as an additive function of each type of input. In Experiments 2 and 3, we evaluated the effects of reducing context retrieval by testing item recovery with free association instructions. In contrast to a cued recall task, in the primed free association task the participant is asked for the first associate to come to mind with each cue, and the study episode is never mentioned. PIER2 predicts that target recovery will be less likely in primed free association because context is not as likely to be retrieved. Its predictions for the effects of backward and otherassociate strengths depend on assigned context weights. Equivalent weights predict that the effects of backward and other-associate strength will be equally reduced by this disruption. Context is weighted equally, so instructions that reduce the retrieval of context should have similar effects on both types of links. Alternatively, if backward but not other-associate strength affects primed free association, differential weights are needed. This issue is important because the findings carry implications for how other associate links manifest their effects. They are more remote from the target than are backward connections, and it may be necessary to recognize this difference by assigning them weaker context weights.

\section{EXPERIMENT 1}

\section{Method}

Participants. A total of 180 undergraduates served as participants in exchange for extra course credit. Thirty were randomly assigned to each of the six between-subjects conditions in replication blocks defined by conditions, lists, and list forms.

Design. The experimental design formed a $3 \times 2 \times 2$ mixedmodel factorial with study disruption (pleasantness rating vs. intentional vs. vowel naming) and test disruption (no disruption vs. 10 min of multiplication) manipulated between subjects. Otherassociate strength (high vs. low) was varied within subjects. Because the same targets were used for strong and weak cues, two forms of each list were prepared. When a target was cued with a strong other-associate cue in Form A, it was cued with a weak otherassociate cue in Form B.

Materials. As is shown in Appendix B, 48 words were selected from the norms to serve as targets for two 24-item study lists (Nelson et al., 1999). An additional 96 words served as test cues, with two cues selected from the associative set of each target. In this manner, the same target was used for each level of cue strength. Each participant was tested on both strong and weak other-associate cues, and different forms of each list (not shown in Appendix B) were used to counterbalance other-associate strength over participants. These cues varied in other-associate strength in accordance with PIER2's Equation A1a, with context weights set to 1.0. Test cues had strong $(M=1.19, S D=.44)$ or weak connections from other associates in the target's set $(M=.26, S D=.17)$. Strong other-associate cues also had more links from other associates in the set than weak cues, averaging $3.52(S D=1.20)$ and $1.65(S D=.56)$ connections, respectively. Target set size averaged $15.13(S D=5.09)$ associates, concreteness rating on a $1-7$ scale averaged $4.53(S D=1.47)$, and printed frequency averaged $25(S D=32)$ times per million words. The associates of these targets had an average of 1.95 links to other associates in the set $(S D=.62)$, and $27 \%$ of the associates produced the target as an associate $(S D=.19)$.

Direct and indirect links joining the test cues and their targets were controlled in all conditions. Forward strength was held constant at weak levels, with targets being produced by their test cues with an average probability of $.02(S D=.03)$. Backward strength was held constant at moderate levels at each level of other-associate strength, with cues being produced by their targets with an average probability of $.14(S D=.07)$. Indirect links involving shared associates and mediators were equated at each level of other-associate strength. Shared associates are associates of both the target and the test cue, and mediators are associates of the test cue that produce the target as an associate. Shared associate strength was low and averaged $.03(S D=.05)$, with an average of $2.39(S D=1.58)$ shared associates. Mediated associate strength was also low and averaged $.003(S D=.01)$, for an average of $.61(S D=1.32)$ mediated connections. Relatively weak cues were used in this experiment to maximize the effects of disruption (see, e.g., Nelson et al., 1998). Other features of the cues that were equated included cue set size, concreteness, and frequency. Test cues produced $14.31(S D=5.48)$ associates in free association, they were rated as moderately concrete at $4.79(S D=1.38)$ on a $1-7$ scale, and they were relatively frequent at 122 per million words $(S D=143)$. During list construction, a program called ListChecker was used to eliminate unintended connections between list items.

Procedure. The participants were tested in individual sessions. Words were presented one at a time during both study and test trials, and each word had to be read aloud when it appeared. The targets were randomly presented in uppercase letters for $3 \mathrm{sec}$ each. Prior to presentation of the study list, a short practice trial consisting of people's first names was shown to familiarize the participants with the procedure. For the disruption-during-study conditions, nondisrupted participants were asked to rate the words for pleasantness or were given intentional learning instructions. Those given pleasantness instructions rated the pleasantness of each target using a 1-5 scale. Those given learning instructions were asked to remember as many words as possible for a later memory test, but they were not told how they would be tested. The participants who were disrupted during study were asked to rapidly name each target's vowels. No reference was made to the upcoming memory test in the pleasantness-rating and vowel-naming conditions.

For the disruption-after-study conditions, the test instructions were read to nondisrupted participants immediately following presentation of the study list. They were told that meaningfully related cues were going to be shown to help them remember the studied words, that the test cues would be presented one at a time, and that they should use each cue to recall a meaningfully related word from the word list just seen. They were also told that, if no studied word came to mind, they could guess. Those assigned to the math disruption condition were given the same instructions after $10 \mathrm{~min}$ of multiple-digit multiplication problems on separate sheets of paper prior to testing. These participants were encouraged to work as rapidly and as accurately as possible on the math. The test phase for both groups was self-paced, and the order of presentation of test cues was independently randomized for each participant.

\section{Results and Discussion}

Analysis of variance (ANOVA). The probabilities of correctly recalling the target as a function of otherassociate strength, disruption during study, and disruption after study are shown in Figure 2. As can be seen, cues with strong input from other associates in the set were generally more effective than those with weak input. This benefit, however, was more evident when disruptions were absent. When attention was disrupted during study by vowel naming or after study by a math task, the relative advantages of stronger other-associate cues were reduced. A three-factor ANOVA confirmed significant effects of other-associate strength, disruption during study, and disruption after study on probability of correct recall $\left[F(1,174)=20.90, M S_{\mathrm{e}}=.010 ; F(2,174)=64.33\right.$, 


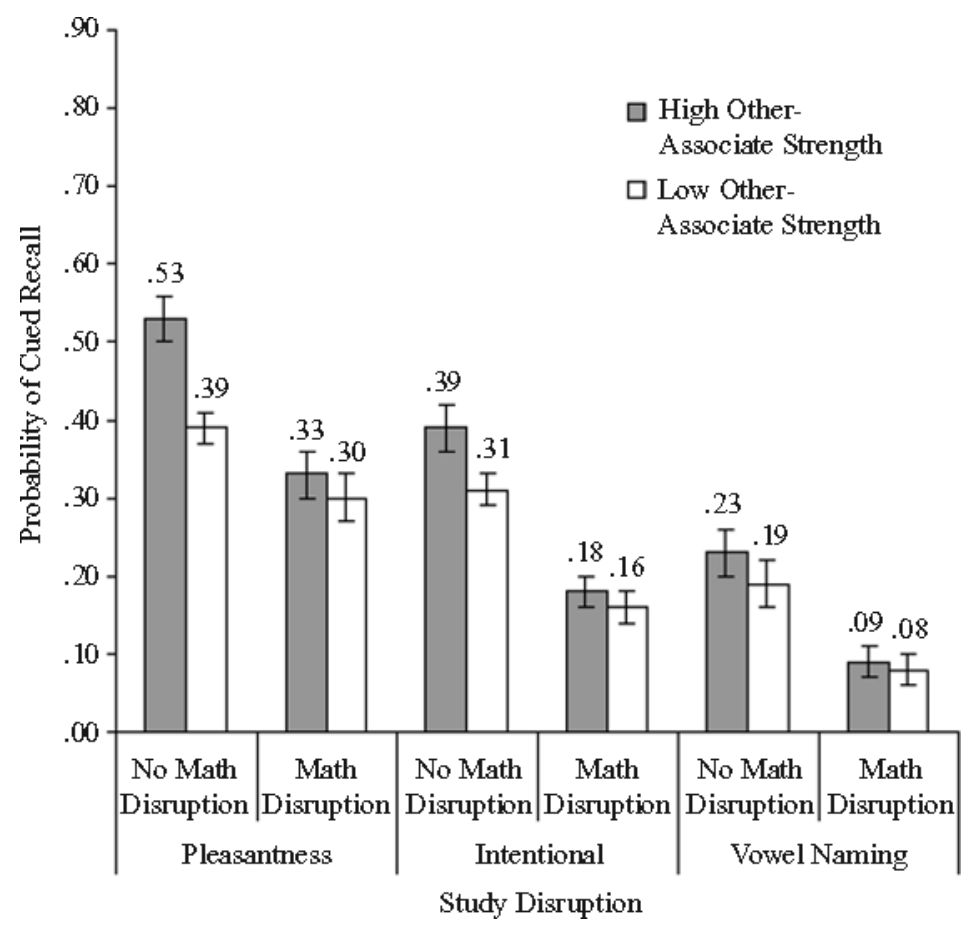

Figure 2. Experiment 1: Probability of cued recall as a function of otherassociate strength, disruption during study, and disruption after study. Error bars reflect standard errors of the means.

$M S_{\mathrm{e}}=.027$; and $F(1,174)=73.71, M S_{\mathrm{e}}=.027$, respectively]. Probability of recall was greater when targets were cued by their stronger (.29) in comparison with their weaker (.24) other-associate cues. Recall was greatest in the pleasantness rating condition (.39), followed by the intentional learning condition (.26), with the lowest level of recall in the vowel naming condition (.15). A Fisher's two-tailed least significant difference (LSD) of .04 showed that the probability of correct recall was significantly different for each condition. The model attributes the superiority of pleasantness ratings over intentional learning to differences in the explicit representation. Such differences have been found before, and the presumption is that pleasantness ratings emphasize individual targets, whereas intentional learning encourages rehearsing the targets together. In the extralist cuing task, transfer-appropriate processing is likely to be greater in the pleasantness condition because the test cue focuses retrieval at this level. Finally, recall was greater when there was no disruption after study (.34) than when $10 \mathrm{~min}$ of multiplication were interpolated between study and test (.19).

Two interaction effects were also reliable. The effects of other-associate strength depended on both disruption during study $\left[F(2,174)=3.35, M S_{\mathrm{e}}=.010, \mathrm{LSD}=.04\right]$ and disruption after study $\left[F(1,174)=7.62, M S_{\mathrm{e}}=.010\right.$, $\mathrm{LSD}=.03]$. The effects of other-associate strength were reliable when the participants focused on meaning or on normal list-learning processes during study but not when processing was disrupted by the need to name vowels. Probabilities of recall were .43 and .35 for pleasantness ratings, .29 and .24 for intentional instructions, and .16 and .14 for vowel naming instructions for the strong and weak other-associate cues, respectively. Similarly, the effects of other-associate strength were significant when attention was not disrupted between study and test, and they were unreliable when it was diverted to the math task. The probabilities of recall for the strong and weak other-associate cues for the nondisrupted test were .38 and .30 , respectively; for the math-disrupted test, they were .20 and .18, respectively. No other effects were significant.

Regression analysis. Because some variables known to affect cued recall correlate naturally, a simultaneous multiple regression analysis was run on the data to determine whether the effects of other-associate strength were the result of such relations (Nelson \& Zhang, 2000). Although these variables were controlled in terms of mean differences, variability in the values may have favored the stronger cues. The regression statistically controls such relations, but the level of evaluation must be changed from the participant level to the item level. The data for each cue-target pairing was scored in each condition for probability of correct recall.

No information about target features was entered into the regression because the same targets were used for implementing the effects of both strong and weak cues. Only other-associate strength and cue related features were entered, including cue frequency and cue set size along 
with forward, backward, mediated, and shared associate strength. An overall analysis pooled over the disruption variables was rejected because attention-switching during and after study reduced the effects of other-associate strength in the subject-based analysis. Instead, separate regressions were completed for each condition to provide a more precise picture of other-associate strength. Data from the intentional learning and pleasantness rating conditions were collapsed into a nondisrupted processing condition. Data from this category were contrasted with the vowel-naming study-disruption condition.

Table 2 shows that other-associate strength was weakly correlated with backward strength $(r=.13)$ and with cue set size $(r=-.22)$. The remaining features were more weakly correlated, indicating that relations with other features known to affect cued recall did not compromise the other-associate strength manipulation. The semipartials revealed similar patterns and magnitudes of correlations with other-associate strength and are not presented. Because the relations among the features were successfully controlled, we expected the regression analyses to lead to the same conclusions as the ANOVA, and this was the case. The $F$ ratios, $M S_{\text {res }}$, adjusted $R^{2}$, standardized betas, and standard errors for each condition are reported in Table 3. As can be seen, other-associate strength affected the probability of cued recall with backward strength and other features controlled, but, as we expected, it significantly affected recall only when performance was not disrupted at all. Importantly, the vowel naming disruption showed a different pattern of beta effects in comparison with the nondisrupted study conditions. Recall probability was especially low after vowel naming, and recall was less dependent on cue-target links and more dependent on cue features (Nelson \& Zhang, 2000).

When the probability of sampling the target given the test cue (PIER2's Equation A3) was substituted as the only predictor for the seven features listed in Table 3 , it explained $25 \%$ of the variance when the participants were not disrupted during either phase, which is $4 \%$ more than what was explained by the seven features. Equation A3 was also a significant predictor of cued recall for those who were disrupted by the math task after study, for which it explained $21 \%$ of the variance. As was expected, the predictive utility of this equation, although significant, declined when study was disrupted by vowel

Table 2

Experiment 1: Correlation Matrix of Feature Variables

\begin{tabular}{lrllllll}
\hline \multicolumn{1}{c}{ Features } & OAS & FS & BS & SAS & MS & QSS QFreq \\
\hline Other-associate strength & \multicolumn{1}{c}{ - } & & & & & & \\
Forward strength & -.14 & - & & & & & \\
Backward strength & .13 & .34 & - & & & & \\
Shared strength & .07 & .05 & .00 & - & & & \\
Mediated strength & -.05 & .40 & .16 & .18 & - & & \\
Cue set size & -.22 & .12 & .06 & -.43 & -.06 & - & \\
Cue frequency & .07 & .02 & -.05 & -.19 & -.02 & .16 & - \\
\hline
\end{tabular}

Note-96 observations were used in these computations. OAS, otherassociate strength; FS, forward strength; BS, backward strength; SAS, shared associate strength; MS, mediated strength; QSS, cue set size; Qfreq, $\log (.5+$ cue frequency $)$. naming, with $13 \%$ and $5 \%$ of the variance explained when the math test was not and when it was presented after study, respectively. The declines in the effectiveness of PIER2's predictions were expected, because context features were least likely to be retrieved in these conditions, particularly when disruptions occurred both during and after study.

The findings will be discussed more fully after the next experiment, but in the overall picture, they confirm the expectations and specific predictions of PIER2. Unconscious input from other associates to the associate used as the test cue makes it more effective, but disrupting the encoding and the retrieval of context reduces this effectiveness.

\section{EXPERIMENT 2}

In Experiment 2, we evaluated the relation between other-associate and backward strength in both cued recall and primed free association. Cued recall instructions require the recovery of context information. Participants are asked to use the test cue to produce a related word from the specific context defined by the study episode. In contrast, free association instructions relax the context requirement by asking only for a related word. No reference to the study list or any specific episode is made and, during testing, both related and unrelated test cues are presented to disguise the relation between study words and test cues. Prior research has shown that the probabilities of target recovery in cued recall and in primed free association are affected by both backward strength and global target connectivity (e.g., Nelson \& Goodmon, 2002; Zeelenberg, Shiffrin, \& Raaijmakers, 1999). Effects of backward strength in the current experiment will replicate past findings, so the effects of other-associate strength are critical. Although global connectivity affects target recovery in these tasks, local connectivity falling only on the associate used as the test cue may not.

As is shown in Table 1, the model predicts that the effects of the two types of links will be governed by the strengths involved in the calculations. Backward strength is determined by a single link from the target, and it can be set high or low, depending on the condition. Although it is straightforward to set low other-associate strength to the level used for low backward strength, high other-associate strength involves multiple links and is likely to exceed the value for high backward strength. As a result, otherassociate strength would seem to be varied over a wider range than backward strength. This difference is not a problem for the model because its predictions are based on summing the strengths multiplied by context weights. Assigning equivalent weights predicts that other-associate strength effects will be greater than backward strength effects because other-associate strength will be varied over a greater range. In contrast, by assigning differentially weaker weights to the more remote other-associate links, the model can predict that other-associate strength effects will be smaller than backward strength effects.

The results of Experiment 2 will determine whether backward and other-associate links should be equally or 
Table 3

Experiment 1: Multiple Regressions With Cue Features and With Equation A3 as Predictors of Probability of Cued Recall for Each Experimental Condition

\begin{tabular}{|c|c|c|c|c|c|c|c|c|}
\hline \multirow[b]{3}{*}{ Statistics } & \multicolumn{8}{|c|}{ Disruption During Study } \\
\hline & \multicolumn{4}{|c|}{ No Disruption } & \multicolumn{4}{|c|}{ Disruption by Vowel Naming } \\
\hline & \multicolumn{2}{|c|}{$\begin{array}{c}\text { No Math Disruption } \\
\text { After Study }\end{array}$} & \multicolumn{2}{|c|}{$\begin{array}{c}\text { Math Disruption } \\
\text { After Study }\end{array}$} & \multicolumn{2}{|c|}{$\begin{array}{c}\text { No Math Disruption } \\
\text { After Study }\end{array}$} & \multicolumn{2}{|c|}{$\begin{array}{l}\text { Math Disruption } \\
\text { After Study }\end{array}$} \\
\hline$F(7,184)$ or $F(7,88)$ & \multicolumn{2}{|c|}{8.04} & \multicolumn{2}{|c|}{11.62} & \multicolumn{2}{|c|}{11.44} & \multicolumn{2}{|c|}{4.45} \\
\hline$M S_{\text {res }}$ & \multirow{2}{*}{\multicolumn{2}{|c|}{$\begin{array}{l}.017 \\
.21^{*}\end{array}$}} & \multirow{2}{*}{\multicolumn{2}{|c|}{$\begin{array}{l}.010 \\
.28^{*}\end{array}$}} & \multirow{2}{*}{\multicolumn{2}{|c|}{$\begin{array}{l}.007 \\
.44^{*}\end{array}$}} & \multirow{2}{*}{\multicolumn{2}{|c|}{$\begin{array}{l}.004 \\
.20 *\end{array}$}} \\
\hline Adjusted $R^{2}$ & & & & & & & & \\
\hline Features & $\beta$ & $S E$ & $\beta$ & $S E$ & $\beta$ & $S E$ & $\beta$ & $S E$ \\
\hline OAS & $.15^{*}$ & .02 & .03 & .01 & .06 & .02 & -.00 & .01 \\
\hline FS & $.23^{*}$ & .40 & $.23 *$ & .31 & $.38^{*}$ & .37 & .21 & .28 \\
\hline BS & $.17 *$ & .14 & $.24 *$ & .11 & $.17^{*}$ & .13 & .10 & .10 \\
\hline SAS & $.24 *$ & .20 & $.32 *$ & .15 & .13 & .18 & .04 & .14 \\
\hline MS & .07 & 1.07 & .12 & .84 & $.26^{*}$ & .99 & $.31 *$ & .76 \\
\hline QSS & .11 & .00 & .09 & .00 & .16 & .00 & -.05 & .00 \\
\hline QFreq & -.12 & .02 & .04 & .01 & $-.20 *$ & .02 & -.14 & .01 \\
\hline$F(1,190)$ or $F(1,94)$ & \multicolumn{2}{|c|}{64.16} & \multicolumn{2}{|c|}{50.14} & \multicolumn{2}{|c|}{15.01} & \multicolumn{2}{|c|}{5.97} \\
\hline$M S_{\text {res }}$ & \multicolumn{2}{|c|}{.016} & \multicolumn{2}{|c|}{.011} & \multicolumn{2}{|c|}{.011} & \multicolumn{2}{|c|}{.005} \\
\hline Adjusted $R^{2}$ & \multicolumn{2}{|c|}{$.25^{*}$} & \multicolumn{2}{|c|}{$.21 *$} & \multicolumn{2}{|c|}{$.13^{*}$} & \multicolumn{2}{|c|}{$.05^{*}$} \\
\hline Equation A3 & $.50 *$ & .04 & $.46^{*}$ & .03 & $.37 *$ & .05 & $.24 *$ & .03 \\
\hline
\end{tabular}

Note- "No disruption" refers to pooled pleasantness rating and intentional learning instructions. Betas are standardized. *Significant at the .05 alpha level. OAS, other-associate strength; FS, forward strength; BS, backward strength; SAS, shared associate strength; MS, mediated strength; QSS, cue set size; Qfreq, $\log (.5+$ cue frequency).

differentially weighted by context. In cued recall, a result showing that other-associate strength has greater effects than backward strength would indicate that both types of links should be equally weighted by context. Regardless of their differences in remoteness from the target, context information is equally well retrieved for each type of link. To the contrary, a result showing that other-associate strength has smaller effects than backward strength despite being manipulated over a broader range would indicate that smaller context weights need to be assigned to other-associate links. The cued recall task requires the retrieval of context, and such information would seem to be less accessible when it is based on input arising from a more remote source in the target's network. In the primed free association task, the test instructions relax the need to recover context, and the model predicts that in this task the effects of both backward and other-associate strength will be reduced in comparison with their effects in the cued recall task. As in cued recall, however, the relative differences in effect size will determine whether equal or different weights should be applied to the two types of links.

\section{Method}

Participants. Twenty-four undergraduates participated in each between-subjects condition, with 12 assigned to each list. They were selected and rewarded as in Experiment 1.

Design. The design formed a $2 \times 2 \times 2$ mixed-model factorial. Test instructions (cued recall vs. free association) were varied between subjects, and both backward strength (high vs. low) and other-associate strength (high vs. low) were varied within subjects.

Materials. Two 24-item lists were constructed by selecting cuetarget pairs from the associative database (Appendix C). In building these lists, we discovered that backward strength and other-associate strength could not be crossed while the target was held constant, so different target words were used. Care was taken to equate target fea- tures known to affect cued recall for each combination of backward and other-associate strength. Across the two lists, target features for set size, concreteness, frequency, connectivity, and resonance averaged $15.98(S D=3.55), 4.70(S D=1.58), 67(S D=86), 2.00$ $(S D=.43)$, and $.58(S D=.15)$, respectively.

All test cues were members of the target's set; that is, no cue had a backward strength of zero according to the norms. Backward strength averaged .30 (SD 5 .06) when it was high and .04 (SD 5 .02) when it was low. Other-associate strength averaged 1.00 (SD 5.28 ) and .04 (SD 5 .04) when it was high and when it was low, respectively. With the summation rule used in PIER2 and with context weights assigned values of 1.0, the absolute values of the strength manipulations show that other-associate strength was varied over a wider range. Net associate strength is the key variable in the model and is defined as the sum of backward and other-associate strength. It averaged $1.29, .34,1.07$, and .10 for strong backward-strong other, strong backward-weak other, weak backward-strong other, and weak backward-weak other, respectively. Other-associate strength was varied over a broader range, but differences in strength for each feature were equated carefully at each level of backward strength.

Direct and indirect cue-target strengths were controlled. All cues had relatively weak preexisting forward links with their targets, with each cue producing its target at an average probability of .09 $(S D=.04)$. For indirect connections, shared associate strength averaged $.02(S D=.02)$ and mediated strength averaged $.01(S D=$ $.01)$. Finally, cue set size was also controlled, and each test cue produced an average of $12.98(S D=4.52)$ associates in free association. ListChecker was used during list development to eliminate unwanted preexisting links within the lists and between the lists, because the cues from both lists were presented in the primed free association task.

Procedure. The procedures used in the extralist cuing task were identical to those used in Experiment 1, including the use of explicit test instructions in which participants were asked to use each cue to recall a related word from the study list. However, the study instructions indicated that we were collecting concreteness ratings (on a $1-5$ scale). In the primed free association task, the study experience was identical to that used in cued recall. In the test instructions, the participants were told that we were now collecting free association norms and that they should produce the first asso- 
ciatively or meaningfully related word to come to mind for each cue. The rating task was never mentioned. Although only one list was studied, cues from both lists were shown and randomly intermixed to discourage intentional retrieval. This procedure also provided a nonstudied baseline in the experiment, for which a smaller sample size was used than was used in the original norms.

\section{Results and Discussion}

Cued recall. Figure 3 presents probability of target recovery as a function of other-associate strength and backward target-to-cue strength. As can be seen, differences in the effects of backward strength and otherassociate strength are apparent. Targets were more likely to be recovered when backward strength was high (.66) than when it was low $\left[.44 ; F(1,23)=45.48, M S_{\mathrm{e}}=.025\right]$, and when other-associate strength was high (.64) than when it was low $\left[.47 ; F(1,23)=23.73, M S_{\mathrm{e}}=.03\right]$. The effects of other-associate strength appeared to be greater when backward strength was high, but the interaction between these two sources was not significant $[F(1,23)=$ $\left.2.73, M S_{\mathrm{e}}=.05\right]$. An omega squared effect size computation indicated that backward strength explained $26 \%$ of the variance, whereas other-associate strength explained $15 \%$. Backward strength had a larger effect even though other-associate strength was varied over a wider range. This finding is theoretically important. Although more remote other-associate links contribute to the effectiveness of the retrieval cue, weaker context weights need to be assigned to this input than to the input provided by the backward links directly activated by the target.

As in Experiment 1, the simultaneous regression analyses at the item level produced results comparable to those

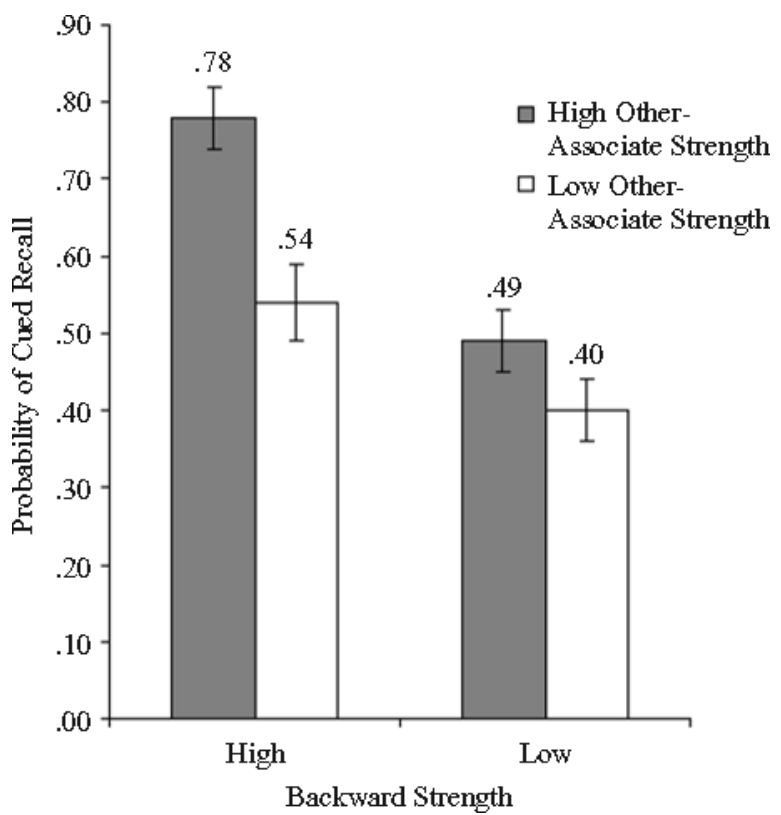

Figure 3. Experiment 2: Probability of cued recall as a function of other-associate strength and backward target-to-cue strength. Error bars reflect standard errors of the mean. found at the subject level. In the first analysis, backward and other-associate strengths were entered as predictors of probability of target recall. The regression was significant $\left[F(2,45)=13.58, M S_{\text {res }}=.04\right]$, with an adjusted $R^{2}$ of .35 . The significant standardized betas were .47 $(S E=.23, t=3.96)$ and $.41(S E=.06, t=3.47)$ for backward strength and other-associate strength, respectively. When the interaction between backward and otherassociate strength was added to the regression, it was not reliable. The next analysis showed that adding other cue and target features known to affect cued recall (Nelson \& Zhang, 2000) did not alter these effects, and none of the added features produced significant betas. This finding indicates that such features were adequately controlled, as was intended.

PIER2's Equation A3, with context weights set to 1.0, was also evaluated as a predictor of cued recall. The mean obtained (and predicted) probabilities of recall were .78 (.76), .54 (.49), .49 (.59), and .40 (.38), respectively, for the strong backward-strong other, strong backward-weak other, weak backward-strong other, and weak backwardweak other conditions. The model's predictions were reasonably accurate, but it overpredicted other-associate strength effects in the weak backward-strong other condition. A regression analysis showed that Equation A3 was a significant predictor $\left[F(1,46)=18.23, M S_{\text {res }}=\right.$ $.05]$, explaining $27 \%$ of the variance of cued recall. This success was comparable to that found in Experiment 1 when there were no disruptions during or after study.

Primed free association. When both tasks were included in an ANOVA, test instructions $[F(1,46)=116.17$, $\left.M S_{\mathrm{e}}=.06\right]$ as well as interactions of test instructions with backward strength $\left[F(1,46)=7.47, M S_{\mathrm{e}}=.03\right.$, $\mathrm{LSD}=.07]$ and with other-associate strength $[F(1,46)=$ $\left.13.12, M S_{\mathrm{e}}=.03, \mathrm{LSD}=.07\right]$ were reliable. As was expected, target recovery was lower in primed free association, but, more importantly, both backward and otherassociate strength effects were reduced in primed free association in comparison with cued recall. Free association instructions reduced target recovery and the effects of both backward and other-associate strengths, as was predicted.

The probabilities of target recovery in primed free association when the targets were (and were not) studied were .22 (.09), .21 (.04), .10 (.03), and .13 (.11) for the strong backward-strong other, strong backward-weak other, weak backward-strong other, and weak backwardweak other conditions, respectively. The nonstudied baseline values corresponded reasonably well with the estimates of forward strength obtained from the free association norms (.09). An analysis of difference scores that took the experimental baseline values into account showed that only backward strength affected primed free association $\left[F(1,23)=8.81, M S_{\mathrm{e}}=.026\right]$. Other-associate strength $\left[F(1,23)<1.0, M S_{\mathrm{e}}=.03\right]$ and the interaction of these variables $\left[F(1,23)=2.00, M S_{\mathrm{e}}=.03\right]$ were not reliable. Although priming was greater when backward strength was high (.15) than when it was low (.05), there 
were no effects on priming when other-associate strength was high (.10) than when it was low (.09).

The results of Experiments 1 and 2 show that associates receiving more input from the target's other associates make stronger cues for recovering the target in cued recall. When accessed by the same target, the activation of other associates in the set strengthen the associate used as the test cue. Whether such strengthening affects recovery, however, depends on whether attention is disrupted during or after encoding and whether the test instructions direct attention to the recent study list. Disrupting memory for general contextual features associated with the setting reduces the influence of other-associate strength. Theoretically, asking participants to name the vowels in the study words disrupts the encoding of context cues more than does asking them to maintain attention to meaning, as when rating pleasantness or when focusing on list learning. Providing free association instructions at test disrupts the retrieval of context information because no reference to the study episode is made.

These results are consistent with the predictions and general expectations of PIER2. In addition, they show that other-associate strength had weaker effects than backward strength in cued recall and virtually no effects in primed free association. A model that assigns the same context weights to these two types of links cannot explain these results. Instead, it appears that more remote associate-to-associate links need to be assigned weaker context weights because such information is less successful in retrieving context information than more directly related backward links. The combination of weaker context weights and an instruction that ignores the importance of context is apparently sufficient to eliminate the benefits produced by other associate strength.

\section{EXPERIMENT 3}

In Experiment 3, we evaluated other-associate strength effects under conditions of primed free association when the target served as the test cue. In terms of Table 1, the target would be used as a test cue for priming the recovery of Associates 3 and 4. PIER2 predicts that, just as associates that receive more input from other associates in the set can be stronger retrieval cues, such items will be more accessible when the target is the test cue. Theoretically, such associates are more strongly activated when the target is studied. However, this prediction is contingent on the context weights assigned to associate-toassociate links. The results of Experiment 2 suggest that, contrary to the context weights of backward links, those assigned to other-associate links tend to be too weak to support priming effects. If this is the case, associates with stronger inputs from other associates in the target's set will not be any more likely to be recovered than those receiving weaker inputs. Higher levels of other-associate strength may be insufficient to support priming effects because the links between implicitly activated associates and context are simply too weak. Interestingly, this in- terpretation assumes that retrieving episodic context is necessary in order for priming effects to be observed.

\section{Method}

Participants. More than the usual numbers of participants were needed to increase sensitivity to small effects, so 120 students participated in exchange for extra course credit. They were randomly assigned to one of the two between-subjects conditions and to one of two separate lists.

Design and Materials. The study experience (pleasantness rating vs. no study) was manipulated between subjects, and otherassociate strength (high vs. low) was varied within subjects. All materials were taken from Experiment 1. During the free association test, 48 cues were shown, including the 24 target words and another set of 24 distractors, which had no associative relationships with the study words. These items were randomly intermixed, and the order was changed for each participant. Distractors were used to bolster the cover story that, in addition to collecting pleasantness ratings, we were also collecting free associations for another experiment. These norms provided baseline values for the cues.

Procedure. The general procedure for the study trial was identical to that used for the nondisrupted pleasantness rating condition of Experiment 1. During the study phase, targets were read aloud and then rated for pleasantness. During the test phase, the targets were presented again with an equal number of intermixed distractors, and the participants were asked to produce, as rapidly as possible, the first word to come to mind that was associatively or meaningfully related. Those assigned to the baseline condition did not study the targets and received only the free association phase of the experiment. The cues were shown at a rate of presentation paced by the participant, and their order was randomly determined for each participant.

\section{Results and Discussion}

The probabilities of targets producing the strong and weak associates in the baseline condition were .18 and .12, respectively. These values closely corresponded to the average values of .15 and .13 found in the large-sample free association database used to construct the lists (Nelson et al., 1999). The probabilities were not very different after pleasantness ratings. The probabilities that the studied target produced the strong and the weak otherassociate strength items were .19 and.13, respectively. With the baseline taken into account, studying the target produced an average priming effect of $1 \%$ for strong and weak associates. An ANOVA that included study condition and other-associate strength as variables indicated that strong other-associate items were more likely to be produced as responses $\left[F(1,118)=36.74, M S_{\mathrm{e}}=.006\right]$, but neither study condition nor the interaction of these variables was significant (both $F \mathrm{~s}<1$ ). A regression analysis with priming calculated for each pair and with other-associate strength as the predictor did not alter this conclusion $\left[F(1,96)<1.0, M S_{\text {res }}=.01\right]$.

Similar results were obtained in several follow-up experiments that included baseline controls. In one, participants were told that we were collecting pleasantness ratings and free associations on the words at the same time. The targets were presented twice, and the participants rated each target's pleasantness on the first presentation and then free-associated to it on its second presentation. In another, the materials of Experiment 3 were used with 
three study trials under intentional learning instructions to strengthen the encoding of context cues. In yet another, the procedures of Experiment 3 were used with concreteness ratings during study, and the test cue consisted of the target plus the first letter of the strong and weak associates. In all of these studies, the results were the same. Relative to baseline controls, associates that received more and stronger inputs from other associates in the target's set were no more likely to be produced than those receiving fewer and weaker inputs. Given hundreds of participants and the replications, the null effects of other-associate strength on priming are unlikely to be due to insensitivity. Levels of other-associate strength that make a target's associate a superior retrieval cue under explicit test instructions are insufficient for making it more accessible as a response in primed free association. Given that backward strength affects primed free association, such results support the hypothesis that context features are linked more weakly to more remote associateto-associate links than to more direct backward target-toassociate links.

\section{GENERAL DISCUSSION}

Research preceding this work indicated that high levels of global connectivity increase target recovery in both cued recall and primed free association tasks. These findings also indicate that the effects of global connectivity are reduced when attention is diverted to a different conceptual task during or after study. Such results bear the hallmarks of a priming phenomenon. A study word recruits its associates during comprehension in a way that provides support for later recall, but disrupting access to context reduces access to this information.

PIER 2 captures these and related findings in a hypothetical target activation process that is sensitive to context (Nelson \& McEvoy, in press). This process assumes that context information related to source is infused throughout the links comprising the studied target's associative network. It multiplies the strength of each preexisting connection in the network by a context weight acquired during the encoding episode. The weighted strengths are then summed to produce net target activation strength as well as the net activation strength for each of the target's associates. The process produces a global index of activation intensity to which the direction of the link in the network is irrelevant. The strengths of target-to-associate and associate-to-associate links are determined by language experience, and the findings of these experiments suggest that the context weights for more remote other-associate links need to be weaker than those for the more directly related target-to-associate links. The context-weighted version of the model attributes the influence of disruptions to failures to encode or to retrieve source information linked to context.

Importantly, PIER2 assumes that activation of the target's associates produces both global and local effects, strengthening both the target and its individual associates. The net activation strength of any individual asso- ciate in the target's set is determined by the sum of the context-weighted inputs from the target and from other associates. Unconsciously activated associates strengthen one another as a function of activating the preexisting links that bind them together. The model predicts that associates with stronger input from the target and from other associates in the target's set make superior retrieval cues for prompting its recall. In consistency with this prediction, the results show that recall was greatest when both backward strength and other-associate strength were high, intermediate when only one of them was high, and lowest when both were low. Regardless of whether the source was from the target or from other associates in the set, recall was more likely when the associate serving as the test cue received more and stronger inputs. These results provide support for PIER2 and other theories, including connectionist models of learning (McClelland \& Rumelhart, 1986) and models of language acquisition (Landauer \& Dumais, 1997).

The results also show that there are limits to mutual strengthening effects in the cued recall task. Local connectivity effects generally follow the same pattern as global effects. Switching attention from the target's meaning and from list-learning processes in order to name its vowels reduces the benefits of other-associate input. Similarly, switching attention from the study task to a multiplication task prior to testing also reduces the benefits. The effects are also reduced when the test instructions refer to general knowledge without mentioning the study episode, as in the primed free association task. Although target-to-associate backward strength affects primed free association, the benefits from other-associate input are eliminated under free association instructions. The benefits evident in cued recall disappeared in primed free association, regardless of whether the effects of otherassociate strength were assessed by cuing target recovery with strong and weak other-associate cues or by using the studied target as a cue for recalling these associates. Because they fail to mention context, free association instructions eliminated the priming effects of recent study produced by other-associate strength but not the effects produced by backward strength. The effects of backward connections were smaller than what was observed in cued recall, but they were clearly apparent.

These findings are important for theory development because they indicate that links among the target's associates contribute less to memory performance than do links from the target to its associates. Other-associate strength was varied over a wider range than backward strength in Experiment 2, but it had a smaller effect on cued recall and no effect on priming. When based on the assumption that backward and other-associate links should be equally weighted, the model's computations incorrectly predict that other-associate strength effects should have been larger than backward strength effects in both cued recall and primed free association. A means for reducing the predicted influence of associate-to-associate links relative to backward links is required, and several ways of modifying the model that preserved its basic in- 
tegrity were considered. The one we chose adopts a version of the context infusion hypothesis that assumes that context weights weaken with distance from the target. However, context infusion is not the only way to explain the findings of these experiments.

A spreading activation hypothesis weights the strength of each associate-to-associate link in the network by the strength of the target-to-associate link (e.g., by multiplying the input from Associate 1 to Associate 3 by the target input to Associate $1[.35 \times .60=.21]$; see Table 1$)$. This weighting procedure is equivalent to using a multiplication rule in place of the addition rule used for Equation A1a in PIER2. Theoretically, activation must spread from the target to its associates and then it must spread to the associate serving as the cue. The spreading activation hypothesis computes local and global connectivity differently, but otherwise, PIER2's computations remain intact. When such computations are applied to the present materials, other-associate strength is manipulated over a narrower range than backward strength $(.05$ and .001 for high and low other-associate strength, respectively). This change allows the model to predict why other-associate strength had smaller effects than backward strength in cued recall and no effect in primed free association. In the implementation of the multiplication rule, backward strengths are unaffected because they are activated directly by the target.

Although the multiplication rule provides a successful explanation of the findings of Experiment 2, it is problematic for several reasons. First, it does not explain why disrupting context during or after encoding in Experiment 1 reduces the influence of other-associate strength. Second, statistical regression research shows that spreading activation rules for computing target activation are not as effective in predicting cued recall as the summation rule (Nelson et al., 2003; Nelson \& Zhang, 2000). The summation rule overpredicts cued recall by an average of only $2 \%$, whereas the proposed multiplication rule underestimates recall by an average of $15 \%$. Third, and more importantly, prior experimental research shows that the effects of global connectivity on target recall are functionally independent of resonant links (Nelson et al., 2003). As with connectivity, words differ in resonance, with some having many and some having few resonant links. When connectivity and resonance are crossed, links of each type benefit remembering but have additive as opposed to interactive effects, suggesting that connectivity effects are not produced by activation spreading back to strengthen the target. Fourth, the multiplication rule is poorly correlated with target connectivitythe very effects that the activation equations (Equations A1 and A1a) were designed to explain. This rule cannot explain the presence of global connectivity effects in cued recall and primed free association, and it follows that it should not be applied to local connectivity effects either.

The context infusion hypothesis was selected because it provides a straightforward explanation of all of our findings. Context is infused throughout the target's associative network, with the strength of the context en- coding decreasing as a function of distance from the target. The target has the largest context weight, backward connections have the next largest, and the smallest weights are assigned to associate-to-associate links, which are more remote. This procedure reduces the potential influence of other-associate strength by changing the context weight, not by changing the preexisting strength of the link between the word pairs in long-term memory. What changes is episodic associate-to-associate strength as determined by multiplying preexisting strength by the context weight. For example, whereas a backward strength of .35 might be assigned a context weight of $.8(.35 \times$ $.8=.28)$, a comparably strong other-associate link might be assigned a weight of $.3(.35 \times .3=.11)$. With weaker context weights, other-associate strength will have smaller effects than backward links in cued recall and in primed free association. The model attributes the weaker effects of other-associate strength to weaker context encoding.

In the cued recall task, the test instructions emphasize the importance of retrieving context, and to the extent that the encoding or retrieval of context is disrupted, other-associate strength effects will be reduced. The model attributes the reductions in other-associate strength effects following the vowel naming and math disruptions to failures to encode or to retrieve context. The disruption produced by vowel naming is important because it indicates that there is an encoding component to the context weights. The effects of context disruptions cannot be attributed to retrieval processes alone. In primed free association, the model attributes the failure to find otherassociate strength effects to the combined influence of weaker context weights and failures to retrieve context. Finally, the model can explain why global connectivity affects primed free association and other-associate strength does not. Other-associate strength is localized to a single associate in the network, which becomes the test cue. Global connectivity was held constant in Experiment 1 by selecting other-associate strength cues for the same target and in Experiment 2 by experimentally controlling it. Global connectivity refers to all associate-to-associate links in the network, and although they may have weaker context links than backward connections, cumulating the weighted sums for all of the associates is apparently sufficient to produce such priming effects. However, no single associate in the network is likely to be strong enough to show priming effects.

The context infusion version of PIER2 assumes that context features are encoded and represented as weights on each link in the target's associative network. As in the original model, preexisting strengths between related pairs of words in the association matrix are strengthened by mutual activation, but they are now differentially weighted by context. The only change lies in the fact that the computation of the target activation process is switched from one based only on free association strengths to one that weights these strengths by context. Context becomes a parameter that must be estimated from the data when none was estimated before, but the remaining computations are unchanged. This change adds complexity that 
makes the model more difficult to test, but it also gives the model a more principled means of explaining why context disruptions have such strong effects on the influence of implicitly activated associates. Disrupting, reinstating, and ignoring context cues have powerful effects on determining whether such associates influence episodic memory. The model becomes more capable of explaining how unconsciously activated information strengthens related information in memory and why the effects produced by such strengthening are so affected by manipulating the encoding and retrieval of information about context.

\section{REFERENCES}

Humphreys, M. S., \& Galbraith, R. C. (1975). Forward and backward associations in cued recall: Predictions from the encoding specificity principle. Journal of Experimental Psychology: Human Learning \& Memory, 1, 702-710.

KINTSCH, W. (1988). The role of knowledge in discourse comprehension: A construction-integration model. Psychological Review, 95, 163-182.

Landauer, T. K., \& Dumais, S. T. (1997). A solution to Plato's problem: The latent semantic analysis theory of acquisition, induction, and representation of knowledge. Psychological Review, 104, 211-240.

McClelland, J. L., \& Rumelhart, D. E. (1986). A distributed model of human learning and memory. In J. L. McClelland \& D. E. Rumelhart (Eds.), Parallel distributed processing: Explorations in the microstructure of cognition (Vol. 2, pp. 170-215). Cambridge, MA: MIT Press.

NeEly, J. H., \& DurgunoǦLu, A. Y. (1985). Dissociative episodic and semantic priming effects in episodic recognition and lexical decision tasks. Journal of Memory \& Language, 24, 466-489.

Nelson, D. L., Bennett, D. J., Gee, N. R., Schreiber, T. A., \& McKinney, V. M. (1993). Implicit memory: Effects of network size and interconnectivity on cued recall. Journal of Experimental Psychology: Learning, Memory, \& Cognition, 19, 747-764.

Nelson, D. L., \& Goodmon, L. B. (2002). Experiencing a word can prime its accessibility and its associative connections to related words. Memory \& Cognition, 30, 380-398.

Nelson, D. L., \& Goodmon, L. B. (2003). Disrupting attention: The need for retrieval cues in working memory theories. Memory \& $\mathrm{Cog}_{-}$ nition, 31, 65-76.

NELSON, D. L., \& MCEvoY, C. L. (in press). Implicitly activated memories: The missing links of remembering. In C. Iaawa \& N. Ohta (Eds.), Learning and memory: Advances in theory and application. Mahwah, NJ: Erlbaum.

Nelson, D. L., McEvoy, C. L., \& Pointer, L. (2003). Spreading activation or spooky action at a distance? Journal of Experimental Psychology: Learning, Memory, \& Cognition, 29, 42-52.

Nelson, D. L., McEvoy, C. L., \& SchreIBER, T. A. (1999). The University of South Florida word association, rhyme, and word fragment norms. Available at http://luna.cas.usf.edu/ nelson/.

Nelson, D. L., McKinney, V. M., Gee, N. R., \& Janczura, G. A. (1998). Interpreting the influence of implicitly activated memories on recall and recognition. Psychological Review, 105, 299-324.

Nelson, D. L., Schreiber, T. A., \& McEvoy, C. L. (1992). Processing implicit and explicit representations. Psychological Review, 99, 322348.

Nelson, D. L., \& Zhang, N. (2000). The ties that bind what is known to the recall of what is new. Psychonomic Bulletin \& Review, 7, 604-617.

RaAiJMakers, J. G. W., \& Shiffrin, R. M. (1981). Search of associative memory. Psychological Review, 88, 93-134.

RatClifF, R., \& MCKoON, G. (1988). A retrieval theory of priming in memory. Psychological Review, 95, 385-408.

Roediger, H. L., III, \& MCDERMOTT, K. B. (1995). Creating false memories: Remembering words not presented in lists. Journal of Experimental Psychology: Learning, Memory, \& Cognition, 21, 803-814.

Tulving, E., \& THOMson, D. M. (1973). Encoding specificity and retrieval processes in episodic memory. Psychological Review, 80, 352373.

UNDERWOOD, B. J. (1965). False recognition produced by implicit verbal responses. Journal of Experimental Psychology, 70, 122-129.

Zeelenberg, R., Shiffrin, R. M., \& RaAijmakers, J. G. W. (1999). Priming in a free association task as a function of association directionality. Memory \& Cognition, 27, 956-961.

\section{APPENDIX A \\ A Computational Example of PIER2's Equations}

\section{Target Activation Process (Equation A1)}

Assume that studying a word primes its representation and the representations of its associates in long-term working memory. Initial self-strength of the target is assigned a nominal value of 1.00 , and remaining values are taken from free association norms. For example, as is shown in the association matrix (see Table A1), the target produces the associates $\mathrm{A}_{1}-\mathrm{A}_{4}$ at probabilities of .60, $.20, .10$, and .10, respectively. Associate 1 produces the target and Associates $\mathrm{A}_{2}-\mathrm{A}_{4}$ at probabilities of $.20, .00, .35$, and .05 , respectively. Equation $\mathrm{A} 1$ assumes that the activation strengths of the target and its associates increase as an additive function of the strength of preexisting links in the network. Targets with more and stronger links to, from, and among their associates are activated to higher levels than those with fewer and weaker links. Similarly, associates with stronger inputs from the target and from other associates in the set are activated to higher levels. Activation self-strengths for the target (Equation A1) and its associates (Equation A1a) are defined and computed as follows, with the results for the associates shown in the bottom row of the matrix. For the sake of simplicity, context weights are set to 1.0 .

$$
\begin{aligned}
\mathrm{S}\left(\mathrm{T}_{i}\right) & =\left[\mathrm{S}(\mathrm{T}, \mathrm{T})+\sum_{i=1}^{n} \mathrm{~S}\left(\mathrm{~A}_{i}, \mathrm{~T}\right)\right]+\sum_{j=1}^{n}\left[\mathrm{~S}\left(\mathrm{~T}, \mathrm{~A}_{j}\right)+\sum_{i=1}^{n} \mathrm{~S}\left(\mathrm{~A}_{i}, \mathrm{~A}_{j}\right)\right] \\
& =[(1.00+.35)]+[(.60+.00)+(.20+.00)+(.10+.80)+(.10+.10)] \\
& =3.25 .
\end{aligned}
$$

$$
\mathrm{S}\left(\mathrm{A}_{i}\right)=\sum_{j=1}^{n}\left[\mathrm{~S}\left(\mathrm{~T}, \mathrm{~A}_{j}\right)+\sum_{i=1}^{n} \mathrm{~S}\left(\mathrm{~A}_{i}, \mathrm{~A}_{j}\right)\right]
$$


so, $\mathrm{S}\left(\mathrm{A}_{3}\right)=\left[\left(.10^{a}\right)+\left(.80^{b}\right)\right]=.90$ and $\mathrm{S}\left(\mathrm{A}_{4}\right)=\left[\left(.10^{a}\right)+\left(.10^{b}\right)\right]=.20$, where $a$ is backward target-to-cue strength and $b$ is other-associate strength.

\section{Cue-Target Intersection Process (Equations A2 and A3)}

In Equation A2, the activation levels of the target and its associates are used in computing the intersection between this information and that activated by the test cue. Forward cue-to-target links and backward targetto-cue links contribute to the signal strength of the target in relation to its test cue (shared associates and mediators were omitted here to simplify the example). To calculate Equation A2 using strong and weak cues, the results of Equation A1 are entered into the top row of the retrieval matrix. For the test cues, normative values for free association strength are entered into the bottom row of the matrix for forward strength, and the selfstrength of the cue is assigned a nominal self-strength of 1.00 .

$$
\mathrm{S}\left(\mathrm{Q}_{j}, \mathrm{~T}_{i}\right)=\sum_{k}^{n} \mathrm{~S}_{j k} \mathrm{~S}_{i k}
$$

Equation A3 is a computation of a signal-to-noise ratio that uses the strengths of competing associates activated by the target and by the test cue as noise elements. Free association norms are used to estimate competitor strengths, and the summed strengths of all competitors determine the noise level. With $q$ and $t$ equal to the competing associates of the test cue and target, respectively, the probability of sampling the target in the presence of cue and target competitors is

$$
\mathrm{P}_{\mathrm{s}}\left(\mathrm{T}_{i} / \mathrm{Q}_{j}\right)=\frac{\mathrm{S}\left(\mathrm{Q}_{j}, \mathrm{~T}_{i}\right)}{\mathrm{S}\left(\mathrm{Q}_{j}, \mathrm{~T}_{i}\right)+\sum_{q}^{n} \mathrm{~S}\left(\mathrm{Q}, \mathrm{A}_{q}\right)+\sum_{t}^{n} \mathrm{~S}\left(\mathrm{~T}, \mathrm{~A}_{t}\right)} .
$$

Example calculations of strong $\left(\mathrm{A}_{3}\right)$ and weak $\left(\mathrm{A}_{4}\right)$ other-associate strength cues are presented in Tables $\mathrm{A} 2$ and $\mathrm{A} 3$, respectively.

Table A1

Association Matrix

\begin{tabular}{lccccc}
\hline \multicolumn{7}{c}{ Association Matrix } \\
\hline Target & A1 & A2 & A3 & A4 \\
\hline Target & 1.00 & .60 & .20 & $\mathbf{. 1 0}$ & $\mathbf{. 1 0}$ \\
Associate 1 & .20 & & & $\mathbf{. 3 5}$ & $\mathbf{. 0 5}$ \\
Associate 2 & .05 & & & $\mathbf{. 3 0}$ & \\
Associate 3 & .05 & & & & $\mathbf{. 0 5}$ \\
Associate 4 & .05 & & & $\mathbf{. 1 5}$ & \\
Net associate strength & 1.35 & .60 & .20 & $\mathbf{. 9 0}$ & $\mathbf{. 2 0}$ \\
\hline
\end{tabular}

Note-A, associate.

Table A2

Retrieval Matrix: Strong Other-Associate Strength Cue $\left(\mathbf{A}_{\mathbf{3}}\right)$

\begin{tabular}{lcccc}
\hline $\begin{array}{l}\text { Cue-Target } \\
\text { Intersection }\end{array}$ & $\begin{array}{c}\text { Primed } \\
\text { Target Word }\left(k_{i}\right)\end{array}$ & $\begin{array}{c}\mathrm{A}_{3} \text { Serving } \\
\text { as Cue }\left(k_{j}\right)\end{array}$ & $\begin{array}{c}\text { Competitors } \\
(t)\end{array}$ & $\begin{array}{c}\text { Competitors } \\
(q)\end{array}$ \\
\hline Target $(i)$ & $3.25^{\mathrm{a}}$ & $.90^{\mathrm{b}}$ & $.20^{\mathrm{e}}$ & - \\
Test cue $(j)$ & $.05^{\mathrm{c}}$ & $1.00^{\mathrm{d}}$ & - & $.30^{\mathrm{f}}$ \\
\multicolumn{5}{c}{$\mathrm{Ss}($ target/strong other-associate cue $)=1.06 /[(1.06)+(.20+.30)]=.68$} \\
\hline
\end{tabular}

Note- atarget self-strength from Equation A1; b primed associate strength derived from Equation A1 a; cforward strength indexed by free association norms; ${ }^{\mathrm{d}}$ test cue nominal self-strength; ${ }^{\mathrm{e}}$ competitor strength for target $(t)$; ${ }^{\mathrm{f}}$ competitor strength for test cue $(q)$.

Table A3

Retrieval Matrix: Weak Other-Associate Strength Cue $\left(\mathbf{A}_{4}\right)$

\begin{tabular}{lcccc}
\hline $\begin{array}{l}\text { Cue-Target } \\
\text { Intersection }\end{array}$ & $\begin{array}{c}\text { Primed } \\
\text { Target Word }\left(k_{i}\right)\end{array}$ & $\begin{array}{c}\mathrm{A}_{4} \text { Serving } \\
\text { as Cue }\left(k_{j}\right)\end{array}$ & $\begin{array}{c}\text { Competitors } \\
(t)\end{array}$ & $\begin{array}{c}\text { Competitors } \\
(q)\end{array}$ \\
\hline Target $(i)$ & $3.25^{\mathrm{a}}$ & $.20^{\mathrm{b}}$ & $.20^{\mathrm{e}}$ & - \\
Test cue $(j)$ & $.05^{\mathrm{c}}$ & $1.00^{\mathrm{d}}$ & - & $.30^{\mathrm{f}}$ \\
\multicolumn{5}{c}{ Ps(target/weak other-associate cue $)=.36 /[(.36)+(.20+.30)]=.42$} \\
\hline
\end{tabular}

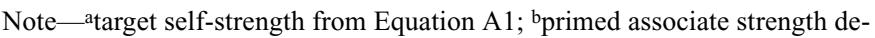

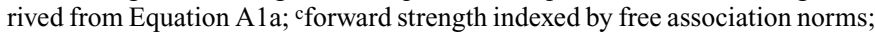
${ }^{d}$ test cue nominal self-strength; ${ }^{\mathrm{e}}$ competitor strength for target $(t)$; ${ }^{\mathrm{f}}$ competitor strength for test cue $(q)$. 
APPENDIX B

Lists Used in Experiments 1 and 3

\begin{tabular}{|c|c|c|c|c|c|}
\hline \multicolumn{3}{|c|}{ List 1} & \multicolumn{3}{|c|}{ List 2} \\
\hline \multicolumn{2}{|c|}{ Associates/Test Cues } & \multirow[b]{2}{*}{ Targets } & \multicolumn{2}{|c|}{ Associates/Test Cues } & \multirow[b]{2}{*}{ Targets } \\
\hline $\begin{array}{l}\text { Strong } \\
\text { OAS }\end{array}$ & $\begin{array}{l}\text { Weak } \\
\text { OAS }\end{array}$ & & $\begin{array}{c}\text { Strong } \\
\text { OAS }\end{array}$ & $\begin{array}{l}\text { Weak } \\
\text { OAS }\end{array}$ & \\
\hline NIGHT & EVENING & AFTERNOON & MAD & ANGER & AGGRAVATE \\
\hline FOOD & DINNER & BANQUET & ARMY & LEADER & COMMANDER \\
\hline LIGHTNING & LOCK & BOLT & CLOTHES & DRESS & COSTUME \\
\hline CAR & DRIVER & $\mathrm{CAB}$ & PROBLEM & TROUBLE & CRISIS \\
\hline QUEEN & HAT & CROWN & CHAIR & SEAT & CUSHION \\
\hline BOOK & CHILD & FAIRYTALE & PRETTY & SWEET & CUTE \\
\hline RICH & GOLD & FORTUNE & MONEY & CENT & DOLLAR \\
\hline DOOR & BAR & HANDLE & FIRE & WARM & FIREPLACE \\
\hline MOUNTAIN & BOOT & HIKING & SLEEP & SWING & HAMMOCK \\
\hline LAUGH & JOKE & HILARIOUS & HAT & COVER & HOOD \\
\hline TOGETHER & CLUB & JOIN & HILL & STEEP & INCLINE \\
\hline ROPE & HOLE & LOOP & SECOND & QUICK & INSTANCE \\
\hline MUSTARD & SANDWICH & MAYONNAISE & TRASH & YARD & JUNK \\
\hline MUSIC & TUNE & MELODY & SICK & DOCTOR & MEDICINE \\
\hline CLEAN & ROOM & MESS & WEAK & MILD & MEEK \\
\hline STAR & SKY & METEORITE & RUN & STILL & MOVEMENT \\
\hline HAPPY & SWING & MOOD & SPAGHETTI & SOUP & NOODLES \\
\hline BUG & BITE & MOSQUITO & SMELL & TEARS & ONION \\
\hline SMELL & ODOR & MUSTY & THOUGHT & FACT & OPINION \\
\hline DOCTOR & RUN & OPERATE & END & RESULT & OUTCOME \\
\hline THIEF & VILLAIN & OUTLAW & PENCIL & WRITE & PAPER \\
\hline PAINT & HOUSE & PAINTER & CAT & FOOT & PAW \\
\hline CATS & ANIMALS & PETS & INSECT & CONTROL & PEST \\
\hline GOD & CHURCH & PRAYER & GAS & JELLY & PETROLEUM \\
\hline
\end{tabular}

Note-OAS, other-associate strength. In Experiment 1, the associates were used as test cues, and the targets were the items to be recovered. In Experiment 3, the targets were studied and were also used as the test cues, and the associates were the items to be recovered. 
APPENDIX C

Lists Used in Experiment 2

\begin{tabular}{|c|c|c|c|}
\hline \multicolumn{2}{|c|}{ List 1} & \multicolumn{2}{|c|}{ List 2} \\
\hline Test Cues & Targets & Test Cues & Targets \\
\hline \multicolumn{4}{|c|}{ High Backward Strength-High Other-Associate Strength } \\
\hline $\mathrm{CHURCH}$ & TEMPLE & DEATH & BURIAL \\
\hline MONEY & WEALTH & KING & MONARCH \\
\hline TOOL & WRENCH & FIRE & BURN \\
\hline BOSS & MANAGER & SPAGHETTI & NOODLES \\
\hline NOUN & PRONOUN & SCHOOL & COLLEGE \\
\hline PANTS & JEANS & AIR & BREATHE \\
\hline
\end{tabular}

High Backward Strength-Low Other-Associate Strength

$\begin{array}{llll}\text { CLIMB } & \text { LADDER } & \text { TICKET } & \text { ADMISSION } \\ \text { STRING } & \text { GUITAR } & \text { HUG } & \text { SQUEEZE } \\ \text { COMFORTABLE } & \text { COZY } & \text { MIX } & \text { COMBINE } \\ \text { HAY } & \text { BARN } & \text { PEOPLE } & \text { PERSON } \\ \text { MARRY } & \text { WED } & \text { COMMENT } & \text { REMARK } \\ \text { WORLD } & \text { UNIVERSE } & \text { GUEST } & \text { VISITOR }\end{array}$

Low Backward Strength-High Other-Associate Strength

\begin{tabular}{llll} 
SAME & EXACT & SQUARE & SHAPE \\
RAIN & THUNDER & BUG & ANT \\
MOVIE & TELEVISION & PENCIL & WRITE \\
TRIP & TRAVEL & GLASS & BREAK \\
UNDERSTAND & KNOW & SICK & COLD \\
PLANT & FLOWER & WAR & FIGHT \\
\multicolumn{1}{c}{ Low Backward Strength-Low } & Other-Associate Strength \\
SECRET & TELL & RESPONSIBILITY & DUTY \\
CHALLENGE & COMPETE & SMILE & TEETH \\
SEW & BUTTON & HANDLE & GRIP \\
DISTANCE & LENGTH & SOME & NONE \\
STRENGTH & MUSCLE & LAYER & BRICK \\
PRESENCE & ABSENCE & MIRAGE & IMAGE \\
\hline
\end{tabular}

(Manuscript received December 19, 2002;

revision accepted for publication December 28, 2003.) 